\title{
Application of Townsend avalanche theory to tokamak startup by coaxial helicity injection
}

\author{
K. C. Hammond ${ }^{1,3}$, R. Raman ${ }^{2,4}$, and F. A. Volpe ${ }^{1}$ \\ ${ }^{1}$ Columbia University, New York, NY 10027 \\ ${ }^{2}$ University of Washington, Seattle, WA 98195 \\ ${ }^{3}$ Present address: Max Planck Institute for Plasma Physics, 17491 Greifswald, Germany \\ ${ }^{4}$ raman@aa.washington.edu
}

\begin{abstract}
Townsend avalanche theory is employed to model and interpret plasma initiation in NSTX by Ohmic heating and coaxial helicity injection (CHI). The model is informed by spatially resolved vacuum calculations of electric field and magnetic field line connection length in the poloidal cross-section. The model is shown to explain observations of Ohmic startup including the duration and location of breakdown. Adapting the model to discharges initiated by CHI offers insight into the causes of upper divertor (absorber) arcs in cases where the discharge fails to initiate in the lower divertor gap. Finally, upper and lower limits are established for vessel gas fill based on requirements for breakdown and radiation. It is predicted that CHI experiments on NSTX-U should be able to use as much as four times the amount of prefill gas employed in CHI experiments in NSTX. This should provide greater flexibility for plasma start-up, as the injector flux is projected to be increased in NSTX-U.
\end{abstract}

\section{Introduction}

Breakdown is the necessary first stage of any plasma discharge. In most tokamak experiments, the primary driver of breakdown is the loop voltage generated by the central solenoid. Stringent restrictions on the loop voltage in the upcoming ITER experiment [1] have motivated extensive studies of breakdown physics over the past thirty years with a view toward increasing the reliability of breakdown while accommodating the restrictions. As part of these efforts, a simple model was developed to describe the requirements on pressure and magnetic field imposed by the loop voltage limitation [2]. Adapted from Townsend avalanche theory [3], the model makes predictions of experimentally testable quantities such as the minimum electric field for breakdown $E_{\min }$ and the breakdown duration $\tau_{\mathrm{bd}}$. While more detailed models and computations are necessary to describe startup scenarios which incorporate auxiliary heating $[4,5]$, the analytical predictions given by the simple model have shown good agreement with experiments on multiple tokamaksincluding DIII-D [2], JT-60U [6], and JET [7]—in the absence of auxiliary heating.

Breakdown is attained in a different way in spherical tokamak (ST) experiments that use transient coaxial helicity injection (CHI) to initiate the plasma $[8,9]$. In this scheme, a large dc bias is placed between the inner and outer walls of the vacuum vessel, which are isolated from one another by narrow gaps in the upper and lower divertors. $\mathrm{CHI}$ is viewed as attractive because of its potential to eliminate the need for the central solenoid, which would in turn increase the feasibility of an ST-based fusion reactor [10]. Recent simulations using the NIMROD code have been able to explain the detailed physics of plasma formation during the $\mathrm{CHI}$ process after the breakdown phase [11], and also describe the conditions under which a large fraction of the injected open magnetic flux could be converted to closed flux [12].

The setup for CHI breakdown differs from that of Ohmic startup both in the mechanism and in the underlying requirements. In terms of the mechanism, $\mathrm{CHI}$ is driven by the electric field resulting from the wall biasing rather than by loop voltage. In addition, while strategies for making breakdown more favorable in the Ohmic case often involve adjusting the poloidal field coil currents to maximize magnetic connection lengths, $\mathrm{CHI}$ has less flexibility in this regard: to drive a given level of plasma current, a minimum amount of poloidal flux must be placed across the divertor [13], thereby setting a effective upper limit on connection length.

The requirements for the location of the breakdown are also more specific in the case of CHI. The breakdown must occur in the lower divertor gap. Parasitic arcing in other locations - particularly the upper divertor gap — must be avoided. Due to the aforementioned lack of flexibility in adjusting the connection lengths, breakdown is typically regulated through careful control of the transient gas pressure: gas is released at the bottom of the vessel just before the beginning of the discharge so that pressure in the lower divertor gap is high while pressure in the upper divertor gap is low.

Given the complexity of the CHI process, modeling of the breakdown conditions would be informative both for evaluating the causes for success or failure of $\mathrm{CHI}$ discharges and for 
determing parameter settings to improve the performance and reliability. To date, however, no modeling of $\mathrm{CHI}$ breakdown has been attempted.

In this paper, we extend the tokamak avalanche theory of Ref. [2] to the CHI breakdown process for the first time and use it to explain observations from both Ohmic and CHI discharges. First, we summarize the main results of the tokamak breakdown model and discuss its applicability to $\mathrm{CHI}$ (Sec. 2). The methodology for comparing the model to NSTX data is described in Sec. 3. In Sec. 4, we test the predictions of avalanche theory against NSTX Ohmic discharges to verify that the model works reasonably well for NSTX. In Sec. 5, we show that analogous calculations for a failed $\mathrm{CHI}$ discharge offer an explanation for the failure. We then employ the theory to establish baseline requirements on gas pressure for planned CHI experiments in NSTX-U, which indicate that a broader space of pressure parameters may be employed than had been used in NSTX.

\section{Avalanche theory}

\subsection{Ohmic breakdown}

The basic model employed in this paper for breakdown in Ohmic discharges is described in detail in Ref. [2], and we provide a brief summary here. A central postulate is that, for a Townsend avalanche to occur, every electron accelerated by the loop voltage must ionize at least one neutral atom prior to hitting the wall. This can be summed up by

$$
L_{c} \geq \frac{1}{\alpha}
$$

where $L_{c}$ is the wall-to-wall connection length along a magnetic field line and $\alpha$, or Townsend's first coefficient, is the average number of ionizing collisions per unit length. In turn, $\alpha$ is given by

$$
\alpha=A p e^{-B p / E},
$$

where $p$ is the gas pressure, $E$ is the electric field, and $A$ and $B$ are gas-dependent constants. For deuterium, $A=$ $510 \mathrm{~m}^{-1} \cdot$ torr $^{-1}$ and $B=1.25 \times 10^{4} \mathrm{~V} \cdot \mathrm{m}^{-1}$ torr $^{-1}$ [14]. From this formula it follows that the minimum Ohmic electric field required for breakdown is

$$
E_{\min }=\frac{B p}{\ln \left(A L_{c} p\right)}
$$

It should be noted that impurity burn-through, the subsequent phase in tokamak start-up [15], may have a more stringent $E_{\text {min }}$ requirement $[7,16]$.

Assuming $E \geq E_{\min }$, one can characterize the avalanche temporally through the differential equation [2]

$$
\frac{\partial n_{e}}{\partial t}=\frac{n_{e}}{\tau_{\mathrm{ion}}}-\frac{n_{e}}{\tau_{\mathrm{loss}}},
$$

where $n_{e}$ is the electron density, the ionization time $\tau_{\text {ion }}=$ $1 / \alpha\left\langle v_{\|}\right\rangle$, and the loss time is $\tau_{\text {loss }}=L /\left\langle v_{\|}\right\rangle$. In turn, $\left\langle v_{\|}\right\rangle$ is the average parallel electron velocity. Thus, this equation considers end losses as the primary loss mechanism and electron-neutral collisions as the primary ionization mechanism. Secondary emission from the walls due to ion impact is neglected.

The mean parallel electron velocity $\left\langle v_{\|}\right\rangle$is estimated in one of two ways depending on $L_{c} \alpha$. If an electron can be expected to ionize many neutrals before being lost (i.e., $L_{c} \alpha \gg 1$ ), it is assumed to travel with a constant average parallel velocity proportional to $E / p$ :

$$
\left\langle v_{\|}\right\rangle=\eta \frac{E}{p},
$$

where $\eta=43$ torr $\cdot \mathrm{m}^{2} \cdot \mathrm{V}^{-1}$ for hydrogen and deuterium. On the other hand, if most electrons "run away," or reach kinetic energies greater than the level associated with the maximum cross-section for electron-neutral collisions, then they are assumed to travel with the average speed of free acceleration by the electric field toward the wall along a field line:

$$
\left\langle v_{\|}\right\rangle=\sqrt{\frac{e E L}{2 m_{\mathrm{e}}}} .
$$

Electrons tend to run away when $E$ is high and $p$ is low, with a threshold ratio of $E / p=(2-2.5) \times 10^{4} \mathrm{~V} \cdot \mathrm{m}^{-1} \cdot \operatorname{torr}^{-1}$ [2]. Integrating Eqn. 4 yields density as a function of time:

$$
n_{e}(t)=n_{e 0} \exp \left(\frac{t}{\tau_{\text {ion }}}-\frac{t}{\tau_{\text {loss }}}\right)
$$

Solving for time and incorporating the appropriate time constants from Eqns. 5 and 6 , the breakdown time $\tau_{\text {bd }}$ can be expressed as

$$
\tau_{\mathrm{bd}}=\left\{\begin{array}{l}
\frac{\ln \left(n_{\mathrm{bd}} / n_{\mathrm{e} 0}\right)}{\eta \frac{E}{p}\left(\alpha-\frac{1}{L}\right)}, \quad E / p \text { below threshold } \\
\frac{\ln \left(n_{\mathrm{bd}} / n_{\mathrm{e} 0}\right)}{\sqrt{\frac{e E L}{2 m_{\mathrm{e}}}}\left(\alpha-\frac{1}{L}\right)}, \quad E / p \text { above threshold }
\end{array}\right.
$$

where $n_{\mathrm{bd}}$ is the density at which the plasma is half-ionized. It is assumed in Refs. [2, 7] that $\ln \left(n_{\mathrm{bd}} / n_{\mathrm{e} 0}\right)=41$ in the absence of pre-ionization.

\subsection{Application to coaxial helicity injection}

In the context of breakdown, $\mathrm{CHI}$ [8] differs from Ohmic startup in two principal ways: (1) the electric field is a DC bias between two coaxial electrodes (i.e. the inboard and outboard walls of the NSTX vacuum vessel), whereas the electric field in Ohmic startup is induced by the changing magnetic flux in the central solenoid; and (2) the electric field is mostly perpendicular to the vacuum magnetic field, whereas the Ohmic field is mostly parallel.

The orbits of electrons in the presence of a strong electric field perpendicular to the magnetic field depend partly on 
the density of the working gas. In a perfect vacuum, electrons will be accelerated along field lines by the parallel component of the electric field, $\mathbf{E}_{\|}$, and will drift with velocity $\mathbf{E} \times \mathbf{B} / B^{2}$. In the presence of significant amounts of working gas, however, elastic collisions with the gas molecules will deflect the electrons from the $\mathbf{E} \times \mathbf{B}$ direction toward the $-\mathbf{E}$ direction by an angle $\theta_{\text {def }}$ such that [17]

$$
\tan \left(90^{\circ}-\theta_{\mathrm{def}}\right)=\lambda / \rho_{\ell}
$$

where $\rho_{\ell}$ is the electron Larmor radius and $\lambda$ is the mean free path for elastic collisions with neutrals. Thus, as the gas density becomes very large $(\lambda \rightarrow 0)$, for example, $\theta_{\text {def }}$ approaches $90^{\circ}$ and the particle travels in the direction of $\mathbf{E}$. In tokamak startup scenarios, however, $\theta_{\mathrm{def}}$ is typically very small. Even under conservative assumptions of $5 \mathrm{eV}$ electrons in a field $B=0.1 \mathrm{~T}$ with deuterium pressure $10^{-3}$ torr, the elastic scattering cross-section is [18] $10^{-19} \mathrm{~m}^{-2}$ and thus $\lambda=10 \mathrm{~cm}$ and $\rho_{L}=75 \mu \mathrm{m}$, leading to $\theta_{\text {def }}=0.04^{\circ}$.

Furthermore, even though $\mathbf{E}_{\perp} \gg \mathbf{E}_{\|}$in the case of $\mathrm{CHI}$, the $\mathbf{E} \times \mathbf{B}$ drift motion itself is small compared to $\left\langle v_{\|}\right\rangle$. The bias between the inner and outer walls is typically $1700 \mathrm{~V}$. In the lower divertor area, where the separation across the gap is as little as $5 \mathrm{~cm}$, the electric field is thus on the order of $3.4 \times 10^{4} \mathrm{~V} / \mathrm{m}$. With $B \approx 0.6 \mathrm{~T}$ in that location, this implies an $\mathbf{E} \times \mathbf{B}$ speed of $5.7 \times 10^{4} \mathrm{~m} / \mathrm{s}$. On the other hand, $\left\langle v_{\|}\right\rangle$ driven by $\mathbf{E}_{\|}$is typically of order $10^{7} \mathrm{~m} / \mathrm{s}$. Assuming a 10 $\mathrm{m}$ connection length (typical for the area around the lower divertor where $\mathbf{E}_{\perp}$ is the strongest), this means that in the time it would take to drift from wall to wall along the field line, an electron would drift upward by $6 \mathrm{~cm}$, which is small on the scale of the NSTX vessel.

When determining minimum pressure requirements for CHI, it is instructive to multiply both sides of Eq. 3 by $L_{c}$ to yield the minimum voltage (across the field line trajectory) for breakdown:

$$
V_{\min }\left(L_{c} p\right)=\frac{B L_{c} p}{\ln \left(A L_{c} p\right)}
$$

This, in effect, is the Paschen curve, as is commonly used to describe dc glow discharges [3, 19]. According to Eq. 10, the denominator vanishes at $L_{c} p \approx 2$ torr $\cdot \mathrm{mm}$, implying that breakdown is not possible at any voltage when $L_{c} p \leq 2$ torr . $\mathrm{mm}$.

It should be noted that the usual expression for the Paschen curve for dc breakdown between two parallel plates includes an additional term in the denominator [19]:

$$
V_{\min }\left(L_{c} p\right)=\frac{B L_{c} p}{\ln \left(A L_{c} p\right)-\ln [\ln (1+1 / \gamma)]}
$$

Here, $\gamma$ is Townsend's second coefficient. Defined as the number of electrons produced per ion through mechanisms other than impact ionization, it accounts for effects such as secondary electron emission due to ion bombardment of the cathode. These effects are necessary for an avalanche to occur between parallel plates. Without an upstream source of electrons that increases in proportion to the number of impact ionization events downstream, the electrons will simply be depleted (for $\gamma=0$ ) or a low, steady-state "dark current" will be maintained (for small but finite $\gamma$ ).

In contrast, secondary electron emission from the wall is not necessary for Ohmic startup in a tokamak. In regions of low poloidal field, field line trajectories will revolve around the torus many times with little change in their poloidal $r$ and $z$ coordinates. Hence, cross-field displacements due to elastic collisions with neutrals will tend to place electrons to points far upstream (and downstream) along the trajectory by nearmultiples of $2 \pi r$. Hence, cross-field displacements resulting from collisions act as an effective upstream electron source. It is for this reason that the condition for avalanche formation in a tokamak (Eq. 1) is independent of secondary electron emission.

The minimum voltage condition in Eq. 11 for parallel plates becomes identical to the minimum condition for tokamaks in Eq. 10 if the second term in the denominator is zero; i.e., for $\gamma \approx 0.58$. Hence, under conditions for which Eq. 1 holds, the upstream-displacing collisions match the effect of a cathode which emits a 0.58 secondary electrons per incident ion.

The effective $\gamma$ due to upstream displacement presumably diminishes as the poloidal displacement of the field line trajectories per revolution increases and/or the total number of toroidal revolutions per trajectory decreases. In the limiting case in which a field line does not make a full toroidal revolution from one wall to the other, as is the case in the divertor gaps in CHI discharges, $\gamma$ is determined primarily by cathode electron emission. Experiments with dc deuterium discharges between parallel plates have exhibited $\gamma \approx 0.05$ [20]. In absence of direct measurements with the NSTX walls, we will use this as an order-of-magnitude estimate for the secondary emission coefficient, although it should be acknowledged that this parameter is known to vary with surface conditions [21].

For the calculations in this paper, we will retain the assumption that $\gamma=0.58$ (i.e., Eq. 1 applies) for field line trajectories in the main NSTX vessel; thus, as mentioned above, $L_{c} p$ must be at least 2 torr.mm for breakdown to be possible. When the possibility for breakdown in the divertor gaps is considered, on the other hand, we will allow for $\gamma \geq 0.05$, in which case i.e., $L_{c} p$ would need to be at least 6 torr $\cdot \mathrm{mm}$.

\section{Spatially resolved calculations}

In previous work, quantities such as $L_{c}$ have been estimated globally to study the relationship with other global parameters such as $V_{\text {loop }}$ and $\tau_{\text {bd }}$. Because $L_{c}$ and $E$ typically vary as a function of the $(r, z)$ location in the vessel cross-section, however, global values of these quantities are ambiguous. $L_{c}$ has been determined a posteriori based on the measured breakdown time, although this exhibited disagreement with $a$ priori estimates of $L_{c}$ [2].

Other previous work has shown the potential for spatiallyresolved calculations such as $L_{c}$ to improve understanding of the breakdown dynamics. In DIII-D, for instance, calcula- 
tions of the work done by the Ohmic field on each electron as it moves along a field line was shown to be maximal near the region where breakdown occurred [22]. Two-dimensional maps of $L_{c}$ have been used to study breakdown in JET [23].

For this work, computations of spatially resolved quantities were based on LRDFIT [24] estimates of the electric and magnetic fields in NSTX. These estimates accounted for induced currents in the vessel due to the time-evolving coil fields. In principle, the code can also reconstruct the plasmainduced fields from magnetic diagnostic signals; however, because these are expected to be small at the time of breakdown, and because the correspondingly low signal-to-noise ratios would lead to large errors in the reconstructions, only the vacuum fields were determined. The $2 \mathrm{D}$ field information was subsequently used to calculate magnetic field lines (with the FIELDLINES code [25]) originating from a grid of points in the $(r, z)$ plane.

Once the field line trajectories were known, a number of spatially resolved quantities could be computed. These included the connection length $L_{c}(r, z)$, or the wall-to-wall length of the field line that passes through the vessel crosssection at $(r, z)$. It should be noted that $L_{c}$ is likely overestimated in this work because 3D error fields are neglected.

The mean parallel electric field $E_{\text {mean }}(r, z)$ along the field line trajectory was determined as

$$
E_{\text {mean }}(r, z)=\frac{1}{L_{c}(r, z)} \int_{\mathbf{s}(r, z)} \frac{V_{\text {loop }}(s)}{2 \pi R(s)} d s+\frac{V_{\mathrm{CHI}}}{L_{c}(r, z)}
$$

Here, $\mathbf{s}(r, z)$ denotes the field line trajectory that intersects the cross-section at $(r, z), V_{\text {loop }}$ is the loop voltage induced by the central solenoid as well as time-varying poloidal field coil currents, and $V_{\mathrm{CHI}}$ is the bias between the inboard and outboard walls (which is zero in the case of Ohmic startup).

This quantity, in turn, may be normalized to the minimum electric field required for breakdown (Eq. 3):

$$
E_{\text {rel }}(r, z)=\frac{E_{\text {mean }}(r, z)}{E_{\min }(r, z)}
$$

$E_{\text {rel }}$ is taken to be zero where breakdown is not possible at any field (i.e., $E_{\min }<0$ ). Correspondingly, the larger $E_{\mathrm{rel}}(r, z)$, the more favorable the conditions are for breakdown at $(r, z)$. In this way, the quantity in Eq. 13 is similar to the work $\left(E_{\text {mean }} L_{c} e\right)$ done on each electron by the Ohmic field. The latter quantity was used as a metric in Ref. [22]. $E_{\text {rel }}$ differs, however, in that it takes the gas pressure into account through $E_{\min }$.

Finally, the breakdown time $\tau_{\mathrm{bd}}$ may be evaluated on the $(r, z)$ grid using the expressions for $E_{\text {mean }}(r, z)$ and $L_{c}(r, z)$.

For Ohmic discharges, the pressure $p$ for these calculations may be determined with good accuracy from ionization gauge measurements prior to breakdown. For CHI discharges, determining $p$ is less straightforward. This is due to the transient nature of the pressure at breakdown in the CHI case. Specifically, the vessel is kept at ultrahigh vacuum until just before the plate energization, at which point gas is released from a plenum into the lower divertor area. For this reason, pressure is expected to vary with height in the vessel and to change rapidly with time.

For the calculations presented in this paper, unless otherwise noted, $p$ throughout the vessel was the ultimate pressure the chamber would have reached after the supplied gas became uniformly distributed. For times less than $5 \mathrm{~ms}$ after the release of the gas, this is expected to be an overestimate for the upper part of the vessel and an underestimate for the lower part of the vessel, particularly in the lower divertor area.

\section{Modeling Ohmic startup}

\subsection{Breakdown time}

Typical Ohmic startup scenarios in NSTX present an additional complication to the breakdown analysis because the magnetic fields are not constant in time. Specifically, the current in the poloidal field coils undergoes a pre-programmed ramp such that the poloidal field null enters the cross-section on the inboard side at a specified time and moves toward the outboard side over the course of a few milliseconds. The maximum of $L_{c}(r, z)$ peaks shortly after the entrance of the null near the central solenoid (CS). This peaking time will be referred to hereafter as the null time, $t_{\text {null }}$.

The null time does not necessarily coincide with the onset of the loop voltage. In particular, for the example Ohmic discharge to be analyzed in this section, $t_{\text {null }}$ occurs $9 \mathrm{~ms}$ later than the onset at $t=0$. During this time, the highest connection lengths increase from $0.6 \mathrm{~km}$ to $>3.5 \mathrm{~km}$. Although the loop voltage is on for times $t \leq t_{\text {null }}$, negligible plasma activity is observed until after $t_{\text {null }}$. Hence, for this discharge, breakdown parameters will be derived using the fields at $t=t_{\text {null }}$.

Breakdown calculations for sample Ohmic discharge 127655 are shown in Fig. 1. Fig. 1a shows the distribution of $L_{c}$ at $t=t_{\text {null }}$, indicating that the greatest connection lengths appear near the CS and slightly below the midplane.

Fig. $1 \mathrm{~b}$ shows the ratio $E_{\text {mean }} / p$ used for determining whether the breakdown time should be calculated in the runaway-electron regime (Eq. 8). The thick dashed line indicates the threshold value of $2.5 \times 10^{4} \mathrm{~V} \cdot \mathrm{m}^{-1} \cdot$ torr $^{-1}$. Since most of the cross-section is in the non-runaway regime, and values of $E_{\text {mean }}$ in the region to the left of the dashed line rarely exceed $30 \%$ of the threshold value, breakdown times for the entire cross-section-even those exceeding the nominal threshold value-were calculated in the non-runaway regime.

Calculated breakdown times for discharge 127655 are shown in Fig. 1c. In portions of the cross-section where breakdown conditions are satisfied, which coincide with regions where $\tau_{\mathrm{bd}}<10 \mathrm{~ms}, \tau_{\mathrm{bd}}$ mostly falls within the range of 2-4 ms.

These results may be compared with direct measurements of the global breakdown time. For Ohmic start-up, this is typically measured as the time between the onset of the loop voltage and the first peak in a reading of $\mathrm{D}_{\alpha}$ emission. However, for the discharge analyzed here, in which the magnetic field varies significantly on the breakdown time scale, we consider 

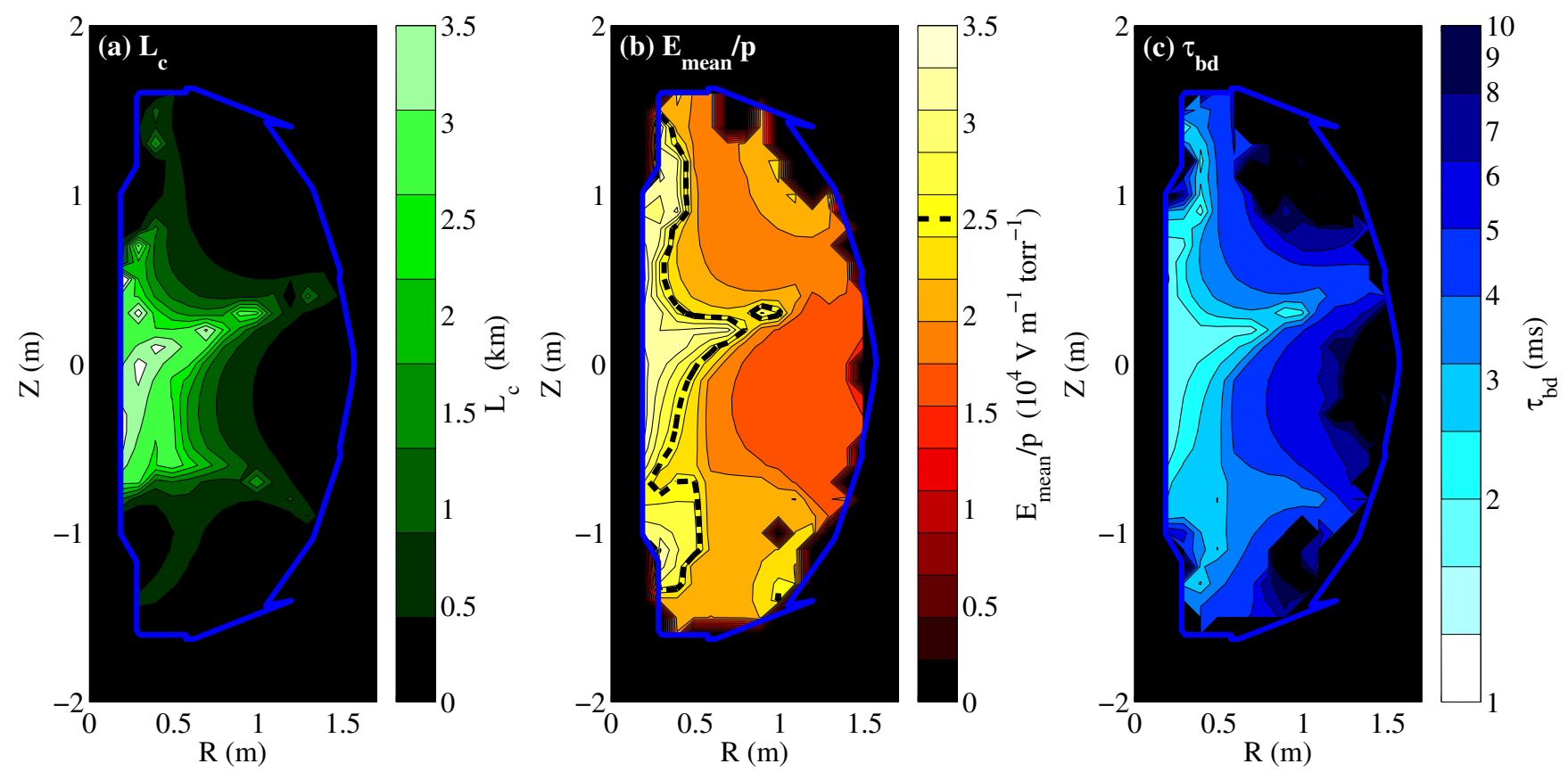

Figure 1: Spatially resolved quantities relevant to breakdown for an example Ohmic discharge in NSTX. All quantities are determined using fields calculated at the null time, $9 \mathrm{~ms}$ after the onset of the loop voltage. (a) Connection length, $L_{c}$. (b) Ratio of electric field to pressure, $E_{\text {mean }} / p$, for determining the collisionality regime under which breakdown time (Eq. 8 ) is estimated. The dashed line is the contour for the threshold value of $2.5 \times 10^{4} \mathrm{~V} \cdot \mathrm{m}^{-1} \cdot \mathrm{torr}^{-1}$. (c) Breakdown time, $\tau_{\mathrm{bd}}$.

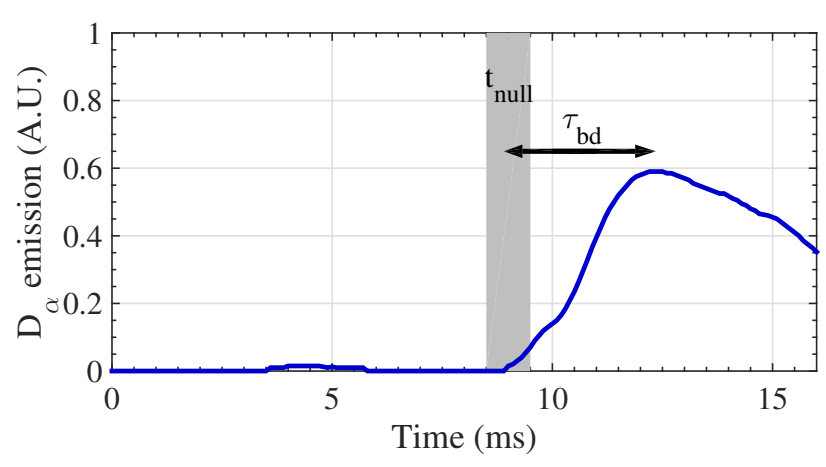

Figure 2: Midplane $\mathrm{D}_{\alpha}$ emission recorded during discharge 127655 .

the null time to be a more appropriate reference time for $\tau_{\mathrm{bd}}$. This is supported by Fig. 2, which shows the signal from a filterscope recording $\mathrm{D}_{\alpha}$ emission from the vessel midplane after the onset of the loop voltage $(t=0)$. Note the neglible emission level prior to $t_{\text {null }}=9 \mathrm{~ms}$. Afterward, the signal rises and reaches an initial peak at $12 \mathrm{~ms}$. The $3 \mathrm{~ms}$ between $t_{\text {null }}$ and the first peak agrees well with the times calculated according to the fields at $t=t_{\text {null }}$ (Fig. 1c). Thus, in spite of the complications to the analysis posed by the experimental conditions in NSTX, the Townsend model appears to produce reasonable predictions for the breakdown characteristics.

\subsection{Predicting the visible breakdown region}

The relative electric field, $E_{\text {rel }}(r, z)$ (Eq. 13) is a measure of the favorability for breakdown in the flux tube at $(r, z)$. A qualitative comparison between $E_{\text {rel }}$ and a fast-camera image of the breakdown of an Ohmic discharge (\#134244) is shown in Fig. 3. In this instance, $E_{\text {rel }}$ (Fig. 3a) is greatest in a region next to the central solenoid, centered slightly below the midplane. The fast-camera image shows a glow that is brightest in a similar region, supporting the notion of $E_{\text {rel }}$ as a predictor of breakdown location. While fast-camera footage was also available for the discharge shown in Fig. 1, the signalto-noise ratio was not sufficient for a clear view of the breakdown phase.

\section{Modeling and projections for $\mathrm{CHI}$ discharges}

$\mathrm{CHI}$ is implemented by driving current along externally produced field lines that connect coaxial electrodes mounted on the electrically isolated inner and outer vacuum vessel components in the presence of externally generated toroidal and poloidal magnetic fields. In NSTX, the inner and outer annular target plates of the poloidal divertor at the bottom of the vacuum vessel serve as the electrodes. A CHI discharge is initiated by first producing poloidal field connecting the injector electrodes with poloidal field coils outside the vessel structure. This poloidal field in combination with the toroidal field produces a helical field line structure in the injector re- 


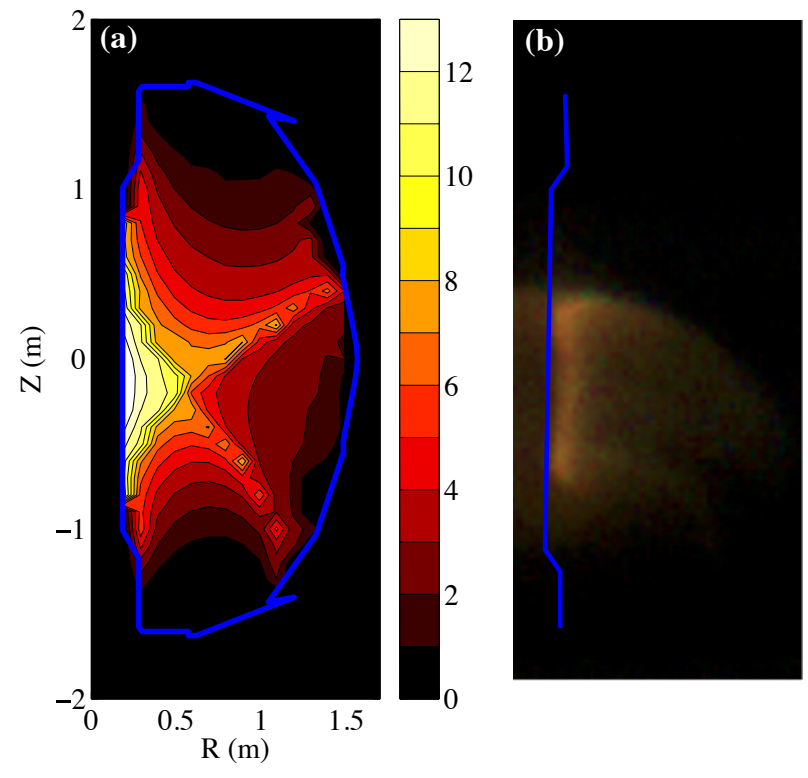

Figure 3: (a) $E_{\text {rel }}$ for the onset of discharge 134244; (b) Fastcamera image of the breakdown of the same discharge with the outline of the central solenoid overlain in blue.

gion. Application of sufficient voltage to these electrodes in the presence of fuel gas causes the gas to break down. The resulting current flows on field lines connecting the electrodes. Increasing the applied voltage increases the injector current. At sufficiently high injector currents, the $\mathbf{J} \times \mathbf{B}$ stress across the current layer exceeds the field line tension of the injector flux, causing the helical current structure to move into the main plasma chamber. In transient CHI, after the injected flux fills the vessel, the external power supply current is reduced to zero, which causes the injected flux to close in on itself producing closed flux surfaces as described in a number of papers $[8,9,26]$.

\subsection{Absorber arcs}

Occasionally during CHI discharges, an arc will occur in the upper divertor gap [8]. Such events are referred to as absorber arcs due to tendency of the magnetic forces to pull the discharge up into the upper divertor rather than out into the vessel. Absorber arcs are deleterious because they emit unwanted impurities and deplete the capacitor banks without contributing to the main plasma.

Absorber arcs on NSTX occur in two forms. The first, and the dominant, form of absorber arcing is initiated when the $\mathbf{J} \times \mathbf{B}$ force that is responsible for injecting the plasma into the main chamber causes this conductive plasma to reach the absorber region. If the plasma contacts the upper divertor plates, then much of the injector current can now flow along the outer vessel walls, bridge the absorber gap along the conductive plasma, and then flow back down along the inner vessel walls. This type of absorber arc is relatively well understood and actively corrected using two methods. First, during transient $\mathrm{CHI}$, the capacitor bank size is chosen so that on the time scale it takes for the plasma to grow and fill the vessel, much of the capacitor bank energy is depleted, so that even if an arc occurs it is weak and does not significantly affect the main plasma discharge. In a second method, which is used in conjunction with proper choice for the capacitor bank size, currents are actively driven in poloidal field coils positioned in the absorber region to actively push the expanding field lines away from the absorber region, as described in Fig. 2 of Ref. [26].

Although most $\mathrm{CHI}$ discharges initiate in the lower divertor (injector) region, in the second, less well-understood form of absorber arcs, there is no breakdown in the injector-instead, on rare occations, the discharge initiates in the absorber. The absence of an arc in the lower divertor is evident from fast camera footage, which normally shows a bright flash in the injector gap [8]. This section discusses this type of absorber arcing.

Two potential mechanisms were considered as possible explanations for these occurrances: (1) unassisted (i.e., no preionization) breakdown in the upper divertor gap due to the dc electric field between the plates and (2) pre-ionizationassisted breakdown in the upper divertor gap resulting from a glow discharge in the main vessel.

To evaluate the first mechanism, an estimate of the connection length $L_{c}$ was required inside the divertor gap. The method of field line tracing, as used in the earlier analysis, was expected to be subject to large errors. This is because the divertor plates contain structural elements that are smaller than the scale of the grid on which the magnetic field is calculated. Hence, instead of determining a spatially resolved model of the divertor gap area, a single representative $L_{c}$ value for the gap area was calculated as

$$
L_{c}=w_{\text {gap }} \frac{B_{\text {t,gap }}}{B_{\text {p,gap }}},
$$

where $w_{\text {gap }}$ is a typical distance between oppositely charged plates in the divertor gap in the $r, z$ plane (ranging from $3 \mathrm{~cm}$ to $7 \mathrm{~cm}$ ), and $B_{\mathrm{t}, \mathrm{gap}}$ and $B_{\mathrm{p}, \mathrm{gap}}$ are the toroidal and poloidal components of the magnetic field, respectively, interpolated at a point in the middle of the gap. Typical values of $L_{c}$ ranged from $2 \mathrm{~m}$ to $6 \mathrm{~m}$.

Typical upper limits on working gas pressure $p$ (as defined in Sec. 3) ranged from 6 to $8 \times 10^{-5}$ torr. For an unassisted breakdown in deuterium to be possible at any voltage, Paschen's law for deuterium indicates that $p L_{c}$ must be at least 2-6 torr $\cdot \mathrm{mm}$ (Sec. 2.2). However, for all of the $\mathrm{CHI}$ shots analyzed in this study, $p L_{c}<0.6$ torr $\cdot \mathrm{mm}$. Considering that $p$ is likely overestimated, unassisted breakdown seems implausible as the mechanism for the absorber arcs.

To evaluate the second candidate mechanism, the feasibility of breakdown within the main vessel was evaluated by calculating $E_{\mathrm{rel}}$ and $\tau_{\mathrm{bd}}$ using the methods described in Sec. 3 for some sample absorber-arc discharges. In this case, conservative estimates of $E_{\text {mean }} / p$ (with $p$ determined as discussed in Sec. 3) exceeded the threshold value of $2.5 \times 10^{4} \mathrm{~V} \cdot \mathrm{m}^{-1}$. torr $^{-1}$ in the entire region in which the breakdown condition $\left(E_{\mathrm{rel}} \geq 1\right)$ was satisfied. In fact, $E_{\text {mean }} / p$ exceeded twice this 
threshold in $70 \%$ of the region. Hence, the calculations of $\tau_{\mathrm{bd}}$ were done in the runaway electron regime.

One example is shown in Fig. 4. The estimates of $\tau_{\text {bd }}$ here are subject to two competing sources of error: the overestimate of $p$ in most of the vessel as discussed in Sec. 3 (which would lead to an underestimate of $\tau_{\mathrm{bd}}$ ), and the application in this discharge of $5 \mathrm{~kW}$ of electron cyclotron heating resonant at $r=0.23 \mathrm{~m}$ (which would tend to accelerate the breakdown). In any case, according to the initial plenum pressure for this discharge, the gas pressure in the upper divertor gap would not have exceeded $7 \times 10^{-5}$ torr, well below the minimum for unassisted breakdown in the gap. This pressure would have permitted breakdown in the main vessel, however, where $L_{c}$ was greater. In particular, the results shown in Fig. 4a-b predict the formation of a bell-shaped plasma $0.14 \pm 0.01 \mathrm{~ms}$ after wall energization. It is not clear from these predictions alone, however, how the bell-shaped plasma in the main vessel would stimulate an arc in the absorber gap.

Fast-camera video of the evolution of this discharge suggests a possible mechanism for initiating the absorber arc. Fig. $4 \mathrm{c}$ shows a fast camera image $0.42 \mathrm{~ms}$ after energization, when the discharge is first visible. In this image, the outline of the discharge resembles the predicted region in Fig. 4a-b. As time progresses, however, the outboard boundary of the glow expands radially outward (Fig. 4c-f). By $0.7 \mathrm{~ms}$, the upper contact point of the discharge has reached the injector gap, and by $0.9 \mathrm{~ms}$ the arc inside the absorber is clearly visible (Fig. 4f). The expansion may be a result of the hoop force acting on the glow discharge current. After the expansion, $\mathbf{E} \times \mathbf{B}$ drift would move the plasma toward the upper divertor.

Hence, the mechanism of a glow discharge pre-ionizing the upper divertor gap to facilitate the absorber arc is supported by calculations and fast-camera observations. Although the conditions were evidently not met in this shot for a breakdown inside the lower divertor gap, the charged walls were able to sustain a breakdown within the main vessel. We note that the origins of these bell-shaped discharges had not been well-understood prior to this study.

\subsection{Gas pressure requirements for $\mathrm{CHI}$ startup}

The gas filling system must strike a balance between competing requirements from the breakdown and ramp-up phases. Initially, the pressure must be high enough in the lower divertor (injector) region to permit breakdown. At the same time, the pressure near the upper divertor (absorber) must not be high enough to cause an absorber arc. (The minimum pressure for breakdown in the absorber is less than the minimum pressure in the injector due to greater $L_{c}$.) Hence, the gas puffed into the injector must be at a high enough pressure to cause breakdown, and this must occur quickly enough to prevent too much neutral gas from accumulating in the absorber. Furthermore, the overall amount of gas admitted to the vessel must not exceed an amount that would cause a radiative collapse.

\subsubsection{Minimum injector pressure}

A lower limit on the peak pressure in the injector gap may be established by assuming that the plenum empties quickly enough so that the $0.1 \mathrm{~m}^{3}$ cavity below the main vessel briefly contains all the gas released from the plenum. (The cavity is connected to the main vessel through the injector gap.) Typical injector connection lengths ranged from 0.5 to $1 \mathrm{~m}$ in NSTX CHI discharges, leading to a minimum requirement of $4 \times 10^{-3}$ torr in the cavity to reach the minimum $p L_{c}$ range of 2-6 torr $\cdot \mathrm{mm}$. This, in turn, implies that the plenum should contain at least 0.6 torr $\cdot \ell$ of gas. Due to the finite time for the plenum to empty ( $4 \mathrm{~ms})$, however, the actual minimum is expected to be higher. Injector breakdown in NSTX was regularly achieved with 2 torr $\ell \ell$ of gas; this may serve as an additional point of reference.

\subsubsection{Maximum absorber pressure}

While unassisted breakdown in the absorber was ruled out as a cause of the absorber arc studied in Sec. 5.1, it is informative to determine a limiting pressure at which such a breakdown could occur. Using the range of 2-6 m calculated for absorber connection lengths during typical NSTX CHI discharges, an upper limit on pressure of $3 \times 10^{-4}$ torr is found. As the NSTX vessel has a volume of approximately $30 \mathrm{~m}^{3}$, this imposes an upper limit of 9 torr $\cdot \ell$.

\subsubsection{Radiation-limiting pressure}

Following a successful breakdown, the plasma undergoes a burn-through phase during which energy losses are dominated by impurity radiation [15]. If a plasma is to be sustained through this phase using only the $\mathrm{CHI}$ mechanism, the radiative losses must be low enough so that they may be sustained by the Ohmic self-heating of the CHI plasma as its current decays. Following burn-through, it is presumed that additional heating sources, such as electron cyclotron resonance heating $(\mathrm{ECRH})$, neutral beam injection (NBI), or Ohmic, would be applied.

The Ohmic self-heating power available can be estimated from typical $\mathrm{CHI}$ parameters. Reconstructions have shown values of poloidal flux $\psi_{\mathrm{p}}$ on the order of 30 mWeber [27]. Since CHI discharges with maximum currents of $I_{\mathrm{p}} \approx 200$ $\mathrm{kA}$ tend to decay with a time constant of roughly $10 \mathrm{~ms}$, the Ohmic heating power density within the plasma volume $V \approx$ $10 \mathrm{~m}^{3}$ is

$$
\begin{aligned}
P_{\text {Ohmic }} & =\frac{I_{\mathrm{p}}}{V}\left(\frac{\Delta \psi_{\mathrm{p}}}{\Delta t}\right) \\
& \approx 60 \mathrm{~kW} \cdot \mathrm{m}^{-3}
\end{aligned}
$$

We estimate the radiatve loss energy by assuming a coronal equilibrium with radiation dominated by a $1 \%$ oxygen impurity population. Under this assumption, the radiated power density is given by [28]

$$
P_{\text {rad }}=0.01 n_{e}^{2} L_{\mathrm{Z}}
$$



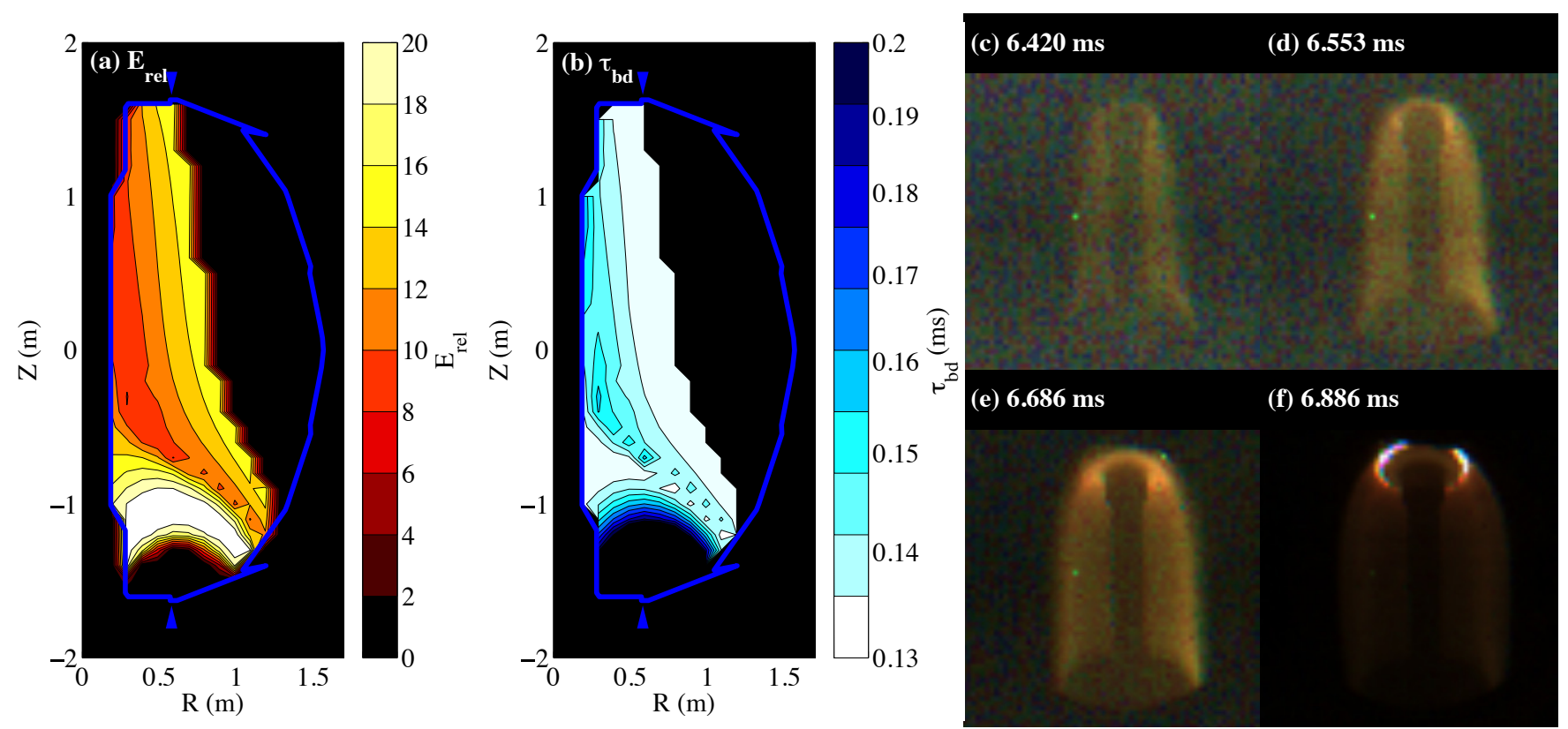

Figure 4: Spatially resolved breakdown calculations (a-b) and fast-camera images (c-f) for a CHI discharge that exhibited an absorber arc but no arc in the injector. (a) Electric field normalized to the minimum for breakdown, $E_{\text {rel }}$. (b) Breakdown time, $\tau_{\mathrm{bd}}$. (c-f) Fast camera frames of the vessel at intervals of $0.42 \mathrm{~ms}, 0.55 \mathrm{~ms}, 0.69 \mathrm{~ms}$, and $0.89 \mathrm{~ms}$ after the plates are energized at $6 \mathrm{~ms}$. In successive images, pixel intensity is amplified by lower factors due to the increasing brightness of the glow; hence, background noise is more prominent in the earlier frames. The blue arrows in (a) and (b) indicate the approximate locations of the injector and absorber gaps on the periphery of the vessel cross-section. Note, however, that $E_{\text {rel }}\left(\tau_{\mathrm{bd}}\right)$ is expected to be substantially lower (higher) in the gaps than in the main vessel due to the lower connection lengths.

in terms of the species- and temperature-dependent constant $L_{\mathrm{Z}}$. Using its peak value for oxygen $\left(9.3 \times 10^{-32} \mathrm{~J} \cdot \mathrm{m}^{3}\right.$ at $\left.T_{e}=18 \mathrm{eV}\right)$ and equating $P_{\mathrm{rad}}$ with $P_{\mathrm{Ohmic}}$ establishes a maximum allowable density of $n_{\max }=8.0 \times 10^{18} \mathrm{~m}^{-3}$. In principle, this limit could be relaxed if auxiliary heating, such as ECRH, were incorporated during the burn-through phase. An additional $500 \mathrm{~kW}$, for example, would raise the maximum density to $1.1 \times 10^{19} \mathrm{~m}^{-3}$. However, depending on which heating method(s) are implemented, the planned NSTX-U $28 \mathrm{GHz}$ system [29] may be limited to the cutoff density of $n_{\text {co }}=9.7 \times 10^{18} \mathrm{~m}^{-3}$.

All three of these limits are at least a factor of two above the typical densities for NSTX CHI plasmas. This is consistent with the routine observation of $30 \mathrm{eV}$ electrons in the discharges: at the lower densities, the $60 \mathrm{~kW} \cdot \mathrm{m}^{-3}$ would have been sufficient to raise the temperature above $18 \mathrm{eV}$ where the radiated power would be maximal for a plasma with oxygen as its main impurity.

The limit on density $n_{\max }$ implies a limit of about 7 torr $\ell$ in the NSTX chamber. An additional $500 \mathrm{~kW}$ of auxiliary power would raise this to 10 torr $\ell$; if the $28 \mathrm{GHz}$ density cutoff is to be obeyed, this would be 9 torr $\ell$. These latter two limits meet or exceed the limit imposed by absorber arc prevention ( 9 torr $\cdot \ell$ ); hence, the addition of ECRH increases the maximum allowable gas fill by $20 \%$ with no further limit imposed by cutoff. With or without ECRH, the upper limit on plenum fill is well above the 2 torr $\ell$ typically used for NSTX CHI shots, indicating that there is considerable flexi- bility to explore larger fills for CHI experiments in NSTX-U. This margin may be important as NSTX-U plans to operate with much higher levels of injector flux in order to increase the current generation potential.

We note that for short, transient plasmas, a time-dependent calculation is needed to correctly calculate the line radiation losses from impurities [30]. In this study, the focus of which is gas breakdown rather than energy balance, and given that we do not know the level of impurities in the plasma or the density and temperature profiles, the simpler steady-state coronal equilibrium is used (but at a temperature that results in the maximum level of radiated power) to obtain order-ofmagnitude estimates for the contribution that radiation losses could play. The calculated result seems consistent with the assumption that for about $1 \%$ oxygen impurity content the measured electron temperature is consistent with the oxygen burn-through in these plasmas.

\section{Conclusions}

In summary, Townsend avalanche theory has been applied to analyze breakdown in Ohmic and CHI discharges in NSTX. By extending the standard 1D Townsend avalanche theory to include spatially resolved calculations, the model is able to provide useful projections for breakdown conditions in more complicated geometries as in CHI discharges. Spatially resolved calculations of breakdown time for an Ohmic dis- 
charge exhibited good quantitative agreement with measurements of $\mathrm{D}_{\alpha}$ emission. Regions with favorable conditions for breakdown were predicted by the distribution of $E_{\mathrm{rel}}$, the ratio of the mean electric field along a field line to the minimum electric field required for breakdown. These regions agree qualitatively with fast camera images of plasma initiation for both Ohmic and CHI discharges. This analysis was also used to help explain the mechanism behind absorber arcs that occur in $\mathrm{CHI}$ discharges in the absence of breakdown in the injector gap.

Finally, accounting for pressure requirements for breakdown in the injector gap, as well as limits imposed by impurity radiation and by the risk of breakdown in the absorber gap, a range of acceptable gas fills of 0.6 to 7 torr. L was established. The upper limit may be raised to 9 torr. $\mathrm{L}$ if 500 $\mathrm{kW}$ of ECRH is incorporated.

The uncertainty in the vessel gas pressure during CHI discharges poses a significant limit on the precision of spatially resolved breakdown analysis for $\mathrm{CHI}$. Nevertheless, the results presented in this paper are promising and may warrant a more detailed modeling of the vessel pressure evolution to permit more accurate quantitative predictions for $\mathrm{CHI}$ discharges in NSTX-U.

Analysis of the upper limits on pressure indicate that the CHI gas fill may possibly be increased by a factor of up to four above typical levels used in NSTX experiments. This flexibility to work with greater particle densities, when coupled with the heating abilities of $\mathrm{ECH}$ and high harmonic fast wave (HHFW) systems [31], has the potential to improve upon the solenoid flux savings achieved in CHI-assisted Ohmic discharges in NSTX [26, 27] and assist with the design of CHI systems in future devices, such as in a ST-FNSF [32].

\section{Acknowledgments}

The authors would like to thank D. Battaglia for the fruitful discussions and assistance with the LRDFIT code. J. Menard and $\mathrm{Z}$. Wang also provided valuable insights for the LRDFIT calculations. In addition, we thank W. Davis for help with the fast camera data. This work was supported through the Oak Ridge Institute for Science and Education (ORISE) Science Graduate Student Research (SCGSR) program, which is managed by Oak Ridge Associated Universities (ORAU) for the US Dept. of Energy (DOE) under contract number DEAC05-06OR23100. This work was also supported by DOE awards DE-FG02-99ER54519 and DE-AC02-09CH11466.

\section{References}

[1] Y Gribov et al Progress in the ITER Physics Basis Chapter 8: Plasma operation and control 2007 Nuclear Fusion 47 S385

[2] Lloyd B, Jackson G, Taylor T, Lazarus E, Luce T and Prater R 1991 Nuclear Fusion 312031
[3] Llewellyn-Jones F 1957 Ionization and Breakdown in Gases (John Wiley \& Sons, Inc.)

[4] Lloyd B, Carolan P and Warrick C 1996 Plasma Physics and Controlled Fusion 381627

[5] Kim H T, Fundamenski W, Sips A and EFDA-JET contributors 2012 Nucler Fusion 52103016

[6] Yoshino R and Seki M 1997 Plasma Physics and Controlled Fusion 39205

[7] de Vries P, Sips A, Kim H, Lomas P, Maviglia F, Albanese R, Coffey I, Joffrin E, Lehnen M, Manzanares A, O’Mulane M, Nunes I, van Rooij G, Rimini F, Stamp $\mathrm{M}$ and JET-EFDA Contributors 2013 Nuclear Fusion 53 053003

[8] Raman R, Jarboe T R, Mueller D, Schaffer M J, Maqueda R, Nelson B A, Sabbagh S A, Ewig R, Fredrickson E D, Gates D A, Hosea J C, Jardin S C, Ji H, Kaita R, Kaye S M, Kugel H W, Lao L L, Maingi R, Menard J, Ono M, Orvis D, Paoletti F, Paul S F, Peng Y K M, Skinner C H, Wilgen J B, Zweben S J and the NSTX research team 2001 Nuclear Fusion 411081

[9] Raman R, Nelson B A, Bell M G, Jarboe T R, Mueller D, Bigelow T, LeBlanc B, Maqueda R, Menard J, Ono M and Wilson R 2006 Physical Review Letters 97 175002

[10] Raman R and Shevchenko V F 2014 Plasma Physics and Controlled Fusion 56103001

[11] Ebrahimi F and Raman R 2015 Physical Review Letters 114205003

[12] Ebrahimi F and Raman R 2016 Nuclear Fusion 56 044002

[13] Jarboe T R 1989 Fusion Technology 157

[14] Rose D J 1956 The Physical Review 104273

[15] Mueller D 2013 Physics of Plasmas 20058101

[16] ITER Physics Basis Expert Group on Disruptions, Plasma Control, and MHD 1999 Nuclear Fusion 39 2577

[17] Heylen A E D 1980 IEEE Proceedings 127222

[18] Engelhardt A G and Phelps A V 1963 Physical Review 1312115

[19] von Engel A 1955 Ionized Gases (Clarendon Press)

[20] Armstrong R J and Bennett T K 1997 Journal of Applied Physics 822147

[21] Korolov I and Donkó Z 2015 Physics of Plasmas 22 093501 
[22] Lazarus E, Hyatt A, Jackson G and Humphreys D 1998 Nuclear Fusion 381083

[23] Maviglia F, Albanese R, de Magistris M, Lomas P, Minucci S, Rimini F, Sips A and de Vries P 2014 IEEE Transactions on Magnetics 507023204

[24] Menard J nstx-u.pppl.gov/software/lrdfit

[25] Lazerson S, Otte M, Bozhnekov S, Biedermann C, Pedersen T S and the W7-X team 2016 Nuclear Fusion 56 106005

[26] Raman R, Mueller D, Nelson B A, Jarboe T R, Gerhardt S, Kugel H W, LeBlanc B, Maingi R, Menard J, Ono M, Paul S, Roquemore L, Sabbagh S, Soukhanovskii V and the NSTX research team 2010 Physical Review Letters 104095003

[27] Raman R, Mueller D, Jarboe T R, Nelson B A, Bell M G, Gerhardt S, LeBlanc B, Menard J, Ono M, Roquemore L and Soukhanovskii V 2011 Physics of Plasmas 18092504

[28] Post D E, Jensen R V, Tarter C B, Grasberger W H and Lokke W A 1977 Steady-state radiative cooling rates for low-density, high-temperature plasmas Atomic Data and Nuclear Data Tables vol 20 (Academic Press, Inc.) pp 397-439

[29] Taylor G, Ellis R A, Fredd E, Gerhardt S P, Greenough N, Harvey R W, Hosea J C, Parker R, Poli F, Raman R, Shiraiwa S, Smirnov A P, Terry D, Wallace G and Wukitch S 2015 EPJ Web of Conferences 8702013

[30] Raman R, Vlases G C and Jarboe T R 1993 Nuclear Fusion 331685

[31] Raman R, Mueller D, Jardin S C, Jarboe T R, Nelson B A, Bell M G, Gerhardt S P, Hooper E B, Kaye S M, Kessel C E, Menard J E, Ono M, Soukhanovskii V and the NSTX Research Team 2013 Nuclear Fusion 53 073017

[32] Menard J E, Brown T, El-Guebaly L, Boyer M, Canik J, Colling B, Raman R, Wang Z, Zhai Y, Buxton P, Covele B, D'Angelo C, Davis A, Gerhardt S, Gryaznevich M, Harb M, Hender T C, Kaye S, Kingham D, Kotschenreuther M, Mahajan S, Maingi R, Marriott E, Meier E T, Mynsberge L, Neumeyer C, Ono M, Park J K, Sabbagh S A, Soukhanovskii V, Valanju P and Wooley R 2016 Nuclear Fusion 56106023 